Economía Teoría y PrÁctica • Nueva Época, número 47, julio-diciembre 2017, pp. 135-166, http://dx.doi.org/10.24275/ETYPUAM/NE/472017/Castaneda

\title{
Análisis de la (re)composición del sistema financiero mexicano en el periodo 2007-2014*
}

\section{Analysis of the (re)composition of the Mexican financial system in the period 2007-2014}

\author{
Ricardo David Castañeda Orozco** \\ y David Juárez-Luna***
}

\begin{abstract}
RESUMEN
Este artículo estudia cómo ha cambiado el sistema financiero mexicano. Para ello se emplean Cadenas de Markov con el objeto de analizar las transiciones ocurridas en el periodo 2007-2014. El resultado principal muestra que, si bien, el número de instituciones financieras se incrementó considerablemente, existe una alta probabilidad de que una institución entrante se constituya como Sofom ENR. La creciente tendencia de incorporación de Sofom ENR, en un marco no regulado, podría implicar algunos riesgos para la población mexicana. Lo que sugiere que se deben contemplar algunas medidas relacionadas a las Sofom ENR como pueden ser: incrementar las restricciones para incorporar estas entidades, fomentar su cambio a figuras reguladas o, ajustar su marco regulatorio para convertirlas en entidades más competitivas y confiables.
\end{abstract}

Palabras clave: Transiciones, Cadenas de Markov, regulación.

Clasificación JEL: G20, G23, G28

\begin{abstract}
This paper studies how the Mexican financial system has changed. We use Markov chains to analyze the transitions the Mexican financial sector has experienced in the period 2007-2014. The main result shows that, although the number of financial institutions increased considerably, a new financial institution is highly likely to adopt the structure of an unregulated multi-purpose financial entity (Sofom ENR). The growing tendency to incorporate as Sofom ENRS, under an unregulated framework, may entail certain risks for the Mexican population. This in turn suggests that the authorities should consider measures relating to Sofom ENRS, such as: increasing restrictions on founding such entities, pressuring them to change to regulated entities, or adapting the regulatory framework to make them more competitive and reliable.
\end{abstract}

Keywords: Transitions, Markov chains, Regulation.

JEL Classification: G20, G23, G28

* Fecha de recepción: 10/06/2015. Fecha de aprobación: 20/02/2017.

** INAI, México. Correo electrónico: rcastaneda3169@hotmail.com. ORCID 0000-0003-45181602

*** Universidad Anáhuac México, campus Norte, México. Correo electrónico: david.juarez@ anahuac.mx. ORCID 0000-0002-8013-699X 


\section{INTRODUCCIÓN}

El uso del sistema financiero formal en México es bajo. La Encuesta Nacional de Inclusión Financiera (INEGI, 2012) ${ }^{1}$ indica que 44 por ciento de la población adulta no cuenta con ningún producto dentro del sistema financiero formal (ahorro, crédito, seguros o previsión para el retiro), mientras que 64.5 por ciento de los adultos no ahorran en instrumentos del sistema. Como consecuencia, en los últimos años el gobierno federal se ha preocupado por fomentar la inclusión financiera, ${ }^{2}$ tomando acciones como son: sofisticación del marco regulatorio; fomento a nuevas figuras financieras y estrategias de acceso; fomento a la entrada de nuevos participantes al sector financiero, entre otras. Ante esta realidad es necesario contar con estrategias adecuadas para medir y analizar la inclusión financiera en México.

De acuerdo con el Libro Blanco de Inclusión Financiera emitido por la CNBV $(2012 c)^{3}$ para el periodo 2007-2012, la inclusión financiera se basa en cuatro pilares: 1) Acceso (penetración geográfica); 2) Uso (disponibilidad de mejores productos en términos de calidad y eficiencia; 3) Protección al consumidor, y 4) Educación financiera para mejorar las capacidades financieras de todos los segmentos de la población. Dicho libro también hace referencia a instrumentos e indicadores para medir y evaluar cada uno de los cuatro pilares tanto en la demanda como en la oferta de servicios financieros.

La medición de la demanda de productos financieros se apoya principalmente en diferentes encuestas como la ENIGH, ${ }^{4}$ ENNVIH, ${ }^{5}$ entre otras (Libro Blanco de Inclusión Financiera (2012c)). No obstante, la medición de la oferta es más compleja debido, al menos en parte, al gran dinamismo de la oferta de servicios financieros. Continuamente ingresan nuevos participantes al sistema financiero así como otros salen. Por lo que surge la necesidad de contar con metodologías sofisticadas que capturen esta dinámica de la oferta.

Por tal, el objetivo de este artículo es analizar la dinámica de la oferta de servicios financieros al estudiar cómo ha cambiado el sistema financiero mexicano. Para ello, se emplean Cadenas de Markov para analizar las transiciones ocurridas en el sector financiero durante el periodo 2007-2014. Lo que permite destacar las fortalezas del sistema a la vez que captura la compleja dinámica del mismo.

\footnotetext{
${ }^{1}$ Instituto Nacional de Estadística y Geografía.

2 Dicha preocupación se hace evidente al plasmar los objetivos para la inclusión financiera en dos Planes Nacionales de Desarrollo, 2007-2012 y 2013-2018.

${ }^{3}$ Comisión Nacional Bancaria y de Valores.

${ }^{4}$ Encuesta Nacional de Ingresos y Gastos de los Hogares.

${ }^{5}$ Encuesta Nacional sobre Niveles de Vida de los Hogares.
} 
Los datos se toman principalmente de fuentes emitidas por la CNBV. Tales fueron el Libro Blanco Relativo a Autorizaciones, Fusiones y Revocaciones de Entidades Financieras (CNBV, 2006-2012) y Boletines Estadísticos (CNBV, 2007-2014: a-d).

El principal resultado muestra que se han dado importantes avances en materia de inclusión financiera a través del fortalecimiento y crecimiento de las Entidades de Ahorro y Crédito Popular; así como con la reciente incorporación de los "Bancos de nicho" al sistema financiero. De hecho, en el periodo 2007-2014 el número de instituciones financieras se incrementó considerablemente pasando de 609 al inicio de 2007 a 4,087 al cierre de 2014, lo que significó un crecimiento promedio anual de 31.25 por ciento. No obstante, la mayoría de las nuevas instituciones financieras se constituyeron como Sofom ENR. ${ }^{6}$ Estas instituciones financieras presentan una probabilidad alta de no cambiar de estado. Al mismo tiempo, existe una alta probabilidad de que una institución entrante se constituya como Sofom ENR. Debido a su escasa regulación y a la poca vinculación con la población de bajos ingresos, la incorporación de estas instituciones al sistema podría implicar algunos riesgos para la población mexicana como son: sobreendeudamiento, mayor oferta de servicios poco competitivos, baja confiabilidad en el sistema, entre otros.

Emplear Cadenas de Markov para describir y analizar las transiciones de las instituciones financieras resultó muy adecuado. Además de permitir analizar la evolución de las transiciones ocurridas en el tiempo, nos ayudan a predecir la composición de instituciones financieras al final del año 2015. ${ }^{7}$ No obstante, las Cadenas de Markov presentan algunas limitantes. Partiendo de la información disponible, no se puede garantizar que las probabilidades de transición sean estacionarias o independientes del periodo en consideración. Además, se considera que la probabilidad de transición está dada por la proporción de instituciones que pasaron de un estado a otro en un año. Esto podría presentar limitaciones adicionales debido a las instituciones que salen del sistema. Otra limitante proviene de la información con la que se dispone. No se tiene información suficiente de las Sofom ENR que han salido del mercado. Por tal, se supone que todos los cambios no identificados corresponden con entradas al sector.

Este trabajo se enmarca en la literatura que estudia al sector financiero mexicano. Una línea de esta literatura aborda la concentración, rentabilidad y eficiencia del sistema bancario en México (ver por ejemplo Rodríguez (2003) y Guerrero y Villalpando (2009)). Otra vertiente se enfoca en la competencia bancaria (ver por ejemplo Avalos y Hernández (2006) y Rivera y Rodríguez (2007) entre otros). Un enfoque intermedio, del que se tuvo conocimiento al haber terminado el análisis

\footnotetext{
${ }^{6}$ Sociedad Financiera de Objeto Múltiple, Entidad No Regulada.

${ }^{7}$ El análisis del presente trabajo se terminó de realizar al inicio del año 2015.
} 
de este artículo, aborda competencia y eficiencia de la industria bancaria mexicana (Castellanos, Del Angel y Garza-García (2016)). Dentro del enfoque de competencia bancaria es destacable el trabajo de Rojas (1997) ya que, al igual que el presente trabajo, emplea Cadenas de Markov como herramienta de análisis. A diferencia del presente trabajo que estudia la (re)composición del sistema financiero, Rojas (1997) estudia la competencia por clientes en la industria bancaria de México asumiendo que los clientes de los bancos se comportan de acuerdo a un proceso de Markov de primer orden. Él encuentra un ligero incremento en la rivalidad en el mercado de colocaciones y una caída significativa en la rivalidad en el mercado de captación de recursos financieros. Son pocos los estudios que abordan la estructura o composición del sistema financiero mexicano. De ellos destacamos dos. Hernández (2010) elabora un diagnóstico sobre los obstáculos que enfrenta el sistema financiero mexicano. Encuentra que son dos los principales obstáculos: débil protección de los derechos de propiedad y el estado de la competencia en el sector financiero. Además, en su diagnóstico, destaca el reducido acceso a servicios financieros en México. Para ello, a diferencia del presente artículo, él se enfoca sólo en el número de sucursales bancarias para evaluar el grado de inclusión financiera: 36 por ciento de los municipios tiene al menos una sucursal bancaria. El 64 por ciento restante carece de esta infraestructura.

El segundo trabajo corresponde a Di Giannatale, López y Roa (2008). Ellas desarrollan un marco conceptual para analizar la interacción entre el sistema financiero y el capital social. Presentan una descripción detallada del sector financiero mexicano. Encuentran que el mismo cuenta con diversas instituciones informales que se basan en las redes sociales (capital social). Concluyen que el desarrollo del sector financiero requiere de un mayor conocimiento de las características de las localidades mexicanas: ambiente socioeconómico, organizacional y de gestión de las instituciones de micro financiamiento. ${ }^{8}$

Cabe destacar que el empleo de Cadenas de Markov no es nuevo en el sector financiero mexicano. Destaca el trabajo de Rojas (1997) mencionado anteriormente. Por mencionar otros ejemplos, Ramírez (2000) analiza la probabilidad de que un cliente se cambie de un banco a otro en un mercado de cuatro instituciones de Banca Múltiple en México durante la década de los 90. Él identifica que a partir de 1995 la competencia entre dichos bancos se incrementó. Por otro lado, García (2009) evalúa las probabilidades de prepago de créditos hipotecarios en carteras bursatilizadas. Con ello analiza el impacto potencial que dichos prepagos pueden tener en la rentabilidad de los tenedores de bonos de las carteras bursatilizadas.

\footnotetext{
${ }^{8}$ Resulta interesante el resumen cronológico que realizan Castellanos, Del Angel y Garza-García (2016) sobre los nuevos participantes en el sistema financiero a partir de la crisis de 1995.
} 
A nuestro mejor entender, éste es el primer esfuerzo por analizar detalladamente, empleando Cadenas de Markov, la composición o (re)composición del sistema financiero mexicano. Otro aspecto novedoso del artículo lo constituyen las fuentes de información que se emplean.

El trabajo se divide en cinco secciones. La primera presenta el marco institucional. La metodología y su aplicación al caso de estudio se presentan en la segunda sección. En la tercera se detallan y analizan las transiciones ocurridas en el sector financiero a la par que se contrastan con los servicios financieros relevantes para la población de bajos ingresos. El futuro de las instituciones financieras se discute en la cuarta sección. En la cinco se presentan las conclusiones. El apéndice describe Cadenas de Markov relevantes.

\section{Marco institucional}

Ante la poca utilización del sistema financiero mexicano, el gobierno federal se ha planteado objetivos para la inclusión financiera, mismos que han quedado plasmados en los Planes Nacionales de Desarrollo (PND). A continuación se da una descripción detallada.

\section{I.1 Las acciones del Plan Nacional de Desarrollo y la inclusión financiera}

En materia de inclusión financiera, el fin general del Plan Nacional de Desarrollo (PND) 2007-2012 se estableció como: "Democratizar el Sistema Financiero salvaguardando la solvencia del sistema en su conjunto". Para ello, se establecieron diversas líneas de acción que incluyen, entre otras: ${ }^{9}$

i. Apoyar el arranque y operación de proyectos productivos familiares mediante asesoría y programas de micro financiamiento.

ii. Enfocar las acciones de la banca de desarrollo a la atención de la población en sectores prioritarios que se encuentran desatendidos por el sector financiero privado.

iii.Promover la competencia en el sector financiero a través de la entrada de nuevos participantes, así como promover un marco regulatorio sólido en el sector.

${ }^{9}$ Plan Nacional de Desarrollo 2007-2012. 
Particularmente con relación al punto iii) anterior, se destaca que en el mismo periodo se presentaron ajustes relevantes para el sector en materia regulatoria, como son:

a. El fin de la autorización de operación para las Sociedades Financieras de Objeto Limitado (Sofol).

b. La banca de desarrollo y los fondeadores en general han incrementado sus requerimientos para otorgar líneas de fondeo, lo que ha implicado la necesidad de saneamiento y mejora, particularmente para las Sociedades Financieras de Objeto Múltiple No Reguladas (Sofom ENR).

c. Se concluyó el periodo de gracia para que las Sociedades Cooperativas de Ahorro y Préstamo (SCAP) presentaran su solicitud de autorización ante la Comisión Nacional Bancaria y de Valores (CNBV). ${ }^{10}$

d. Se realizaron modificaciones en la Ley de Instituciones de Crédito (Congreso de los Estados Unidos Mexicanos, 2010) y a la Circular Única de Bancos (CNBV, 2013a) para dar origen a un nuevo tipo de instituciones financieras denominadas "Bancos de Nicho", entidades que deben cumplir con los mismos requerimientos de la banca múltiple, pero brindando un abanico acotado de servicios y con menores requerimientos de capital.

Los puntos c) y $d$ ) citados previamente se vinculan directamente con las acciones para la inclusión financiera.

En esta coyuntura, el periodo 2007-2012 quedó marcado por un conjunto de importantes entradas, salidas y cambios de figura en el sector financiero.

Particularmente las entradas al sector financiero y los cambios de figura sugieren diferentes beneficios para las partes involucradas, entre los que se destacan:

i. Desde el punto de vista de la institución, entrar al mercado o cambiar su figura actual le puede permitir ampliar su gama de productos y servicios, incrementando con ello su campo de acción y rentabilidad potencial.

ii. Para efectos de inclusión financiera, la migración de entidades financieras por lo general significa la sofisticación de operación de la entidad y el seguimiento de un marco regulatorio más ordenado. Una transición a una figura mejor regulada vuelve a la entidad más atractiva para funcionar como vehículo para programas gubernamentales y transmisión de apoyo a la población objetivo.

\footnotetext{
${ }^{10} \mathrm{O}$ al menos uno de varios periodos de gracia que ha otorgado la CNBV a las entidades de este tipo que se encontraban en situación de prórroga condicionada.
} 
iii. Para la población, de igual manera, un grupo de instituciones más amplio y mejor regulado le impacta desde diversas perspectivas (acceso a más productos y servicios, mayor competitividad en el mercado, confiabilidad en el sistema, canales de acceso a la educación financiera, entre otras).

El periodo de transición comentado no necesariamente termina con el sexenio. El Plan Nacional de Desarrollo 2013-2018 también incluye objetivos hacia la inclusión financiera similares a los establecidos en el plan previo.

Estos objetivos incluyen acciones como son fomento a la entrada de nuevos participantes en el sector, la competencia efectiva y la participación de instituciones financieras reguladas en sectores estratégicos, ${ }^{11}$ entre otras.

\section{I.2 Alcance}

Se analizan las instituciones que, dadas sus operaciones, son consideradas como más viables para ofrecer los servicios fundamentales para la inclusión financiera: otorgamiento de crédito y captación de ahorro. Las instituciones consideradas son:

- Sociedad Financiera de Objeto Múltiple, Entidad No Regulada (Sofom ENR). Entran en función en 2006. Pueden cumplir funciones de arrendamiento y/o factoraje financiero, así como otorgar créditos para cualquier finalidad y no están autorizadas para captar ahorro. No necesitan ser autorizadas por la Secretaría de Hacienda y Crédito Público (SHCP), no les aplican normas de requerimiento de capital, límites de operación o aprovisionamiento mínimo, entre otras. ${ }^{12}$

- Sociedad Financiera de Objeto Múltiple, Entidad Regulada (Sofom ER). En el momento que una Sofom ENR se vincula patrimonialmente con otra institución de crédito, generalmente bancos, se vuelve una entidad sujeta de supervisión por parte de la CNBV. ${ }^{13}$

- Unión de Crédito (UC). Instituciones crediticias constituidas como S.A. de C.V. La UC está autorizada para otorgar créditos a los socios de la misma y tampoco puede captar ahorro (CNBV, 2013b). Por lo general están conformadas por personas que se dedican a un mismo sector o actividad empresarial, lo que en teoría fortalece su capacidad de evaluar proyectos productivos y tomar

${ }^{11}$ Pymes, el sector agroalimentario, otros sectores como son: minería, turismo y cultura, al igual que proyectos de infraestructura.

${ }^{12}$ SHCP (2006).

${ }^{13}$ Ídem. 
decisiones para el otorgamiento de créditos. La regulación para las UC existe pero es más simplificada que la de otras instituciones.

- Sociedad Cooperativa de Ahorro y Préstamo (SCAP). Su inicio data de los años 50 con la creación de las cajas populares, pasando por algunos ajustes regulatorios hasta la conformación de la figura de la SCAP en 1994. Su marco regulatorio también se ha sofisticado con el tiempo. Una característica particular de las SCAP reside en sus principios sociales; todos sus usuarios son socios y, en principio, se espera que la posible rentabilidad que genere la SCAP sea devuelta a la comunidad donde opera a través de obras públicas. En general pueden brindar los mismos servicios que la Sofom, pero además están habilitadas para captar ahorro y para ofrecer otros servicios. Su marco regulatorio y de operación es más específico y se especializa cuando se incrementa el tamaño de sus activos (CNBV, 2012a).

- Sociedad Financiera Popular (Sofipo). Junto con las SCAP, conforman las Entidades de Ahorro y Crédito Popular. ${ }^{14}$ Están regidas por un marco regulatorio desde su creación. A diferencia de las SCAP, las Sofipo son S.A. de C.V., tienen fines de lucro y atienden a clientes y usuarios en general. Su abanico de servicios es similar al de las SCAP. De igual manera tienen diferentes niveles de operación en función del tamaño de sus activos (CNBV, 2012b).

- Institución de Banca Múltiple. Definida por la CNBV como "un establecimiento que se dedica principalmente a captar recursos del público para realizar operaciones activas de crédito o de inversión", la Banca Múltiple constituye la figura más sofisticada del sistema financiero; su regulación también es la más sofisticada dentro de las establecidas para las instituciones consideradas en el alcance del proyecto.

- Banca de Nicho. Esta figura se formaliza en 2009 con el fin de fomentar una mayor competencia en el sector al flexibilizar los niveles de capitalización aplicables a la Banca Múltiple. ${ }^{15}$

Quedan fuera del alcance entidades que ofrecen servicios financieros de mayor grado de especialización, tales como: Casas de Bolsa, Sociedades de Inversión, Aseguradoras, Afores, etcétera. De igual manera, quedan fuera del alcance instituciones cuyo marco regulatorio es mínimo lo que conlleva, entre otros factores, a

\footnotetext{
${ }^{14}$ Existe otra figura denominada Sociedad Financiera Comunitaria (Sofinco) que también se considera parte de las Entidades de Ahorro y Crédito Popular. En el Libro Blanco de Inclusión Financiera (CNBV, 2012c) se identifican diez Sofinco a octubre de 2012. Sin embargo, estas entidades quedan fuera del alcance debido a que no hay información específica sobre ellas en los Boletines Estadísticos de la CNBV.
}

${ }^{15}$ Libro Blanco de Inclusión Financiera (CNBV, 2012c). 
una menor disponibilidad de información confiable de las mismas (Asociaciones Civiles, Instituciones de Asistencia Privada-IAP, entre otras). No obstante, se contemplan los casos en los que instituciones de este tipo tienen una transición a alguna de las figuras listadas dentro del alcance.

\section{Metodología}

La (re)composición del sistema financiero es en sí un sistema dinámico debido a la constante entrada y salida de participantes. Esta dinámica se ha visto intensificada debido a las diferentes reformas a la Ley de Instituciones de Crédito que han reducido las barreras a la entrada. ${ }^{16} \mathrm{El}$ proceso de entradas y salidas al sistema se asume como un proceso estocástico. Particularmente, nos apoyamos en las Cadenas de Markov para describir y analizar la evolución de su estructura en el tiempo. Para tal efecto se consideran las transiciones suscitadas en el sector financiero durante el periodo 2007-2014.

\section{II.1 Cadenas de Markov}

Un proceso de Markov es un proceso estocástico donde el último valor de una variable es lo único relevante para estimar el futuro (Kemeny y Snell, 1976). Para una mejor descripción de las Cadenas de Markov seguimos a Ross (1996). Considere una variable aleatoria $S=\left\{s_{n}, n=0,1,2, \ldots\right\}$ que toma valores de un conjunto finito o contable posible. A reserva de que se especifique otra cosa, el conjunto de posibles valores del proceso se denota por el conjunto de enteros positivos $\{0,1,2, \ldots\}$. La variable aleatoria sigue un proceso de Markov si existe una probabilidad fija, denotada por $p_{i j}$, de que al encontrarse en el estado $i$ en el periodo $n, s_{n}=i$, se encuentre en el estado $j$ en el periodo siguiente, $s_{n+1}=j$. Es decir, suponemos que:

$$
P\left(s_{n+1}=j \mid s_{n}=i, s_{n-1}=i_{n-1}, \ldots, s_{1}=i_{1}, s_{0}=i_{0}\right)=p_{i j}
$$

Para todos los estados $i_{0}, i_{1}, \ldots, i_{n-1}, i, j$ y todos los periodos $n \geq 0$. A dicho proceso estocástico se le conoce como una Cadena de Markov. La ecuación anterior puede ser interpretada como sigue: para una Cadena de Markov, la distribución condicional de cualquier estado futuro, $s_{n+1}$, es independiente de los estados pasados, $s_{0}, s_{1}, \ldots, S_{n-1}$, y sólo depende del estado presente, $s_{n}$. A esto se le llama $l a$

\footnotetext{
${ }^{16}$ Ver Castellanos, Del Angel y Garza-García (2016).
} 
propiedad de Markov. Dado que las probabilidades son no negativas y que el proceso describe una transición hacia un estado, tenemos que:

$$
p_{i j} \geq 0, \quad i, j \geq 0, \quad \sum_{j=0}^{\infty} p_{i j}=1, \quad i=0,1, \ldots
$$

Las Cadenas de Markov se representan a través de matrices de transición y de diagramas de transición. Sea $P$ que denota la matriz de probabilidades de transición de un estado a otro, $p_{i j}$, por lo tanto

$$
P=\left[\begin{array}{cccc}
p_{00} & p_{01} & p_{02} & \cdots \\
p_{10} & p_{11} & p_{12} & \cdots \\
\vdots & & & \\
p_{i 0} & p_{i 1} & p_{i 2} & \cdots \\
\vdots & \vdots & & \vdots
\end{array}\right]
$$

Algunas particularidades a considerar:

- Una Cadena de Markov es regular si la matriz de transición $P$ o alguna de sus potencias tiene únicamente entradas estrictamente positivas.

- Partiendo de un conjunto de estados llamado estado inicial (denotado por $S_{O}$ ), la aplicación de una Cadena regular llevará eventualmente a un estado estable. En este contexto, un estado estable implica que: y así sucesivamente.

- Una cadena regular llevará a un estado en el que se cumple que: $S_{0} \boldsymbol{P}=S_{1} ; S_{1} \boldsymbol{P}=S_{2} ; S_{2} \boldsymbol{P}=S_{3} ;$ y así sucesivamente.

- Una cadena regular llevará a un estado $S_{n}$ en el que se cumple que: $S_{n} \boldsymbol{P}=S_{n}$.

\section{II.2 Cadenas de Markov y el sistema financiero mexicano}

Para el presente análisis el conjunto de estados $\mathrm{S}=\{\mathrm{N}, \mathrm{R}, \mathrm{U}, \mathrm{C}, \mathrm{F}, \mathrm{B}, \mathrm{M}, \mathrm{K}\}$ representa los diferentes tipos de instituciones financieras descritas anteriormente, donde:
N: Sofom ENR.
R: Sofom ER.
U: Unión de Crédito.
C: SCAP.
F: Sofipo.
B: Banco de nicho.
M: Banca Múltiple.
$\mathrm{K}$ : Representa la entrada o salida del mercado. 
El estado $K$ captura el hecho de que el capital que sale del sistema, por ejemplo ante la escisión de un Banco $(M)$, podría utilizarse para conformar otra figura financiera, por ejemplo una Sofom ENR $(N)$. Asimismo, una entidad que sale del sector podría volver a entrar en periodos posteriores. ${ }^{17}$ Además, el estado $K$ simplifica el análisis al agrupar tanto las entradas como las salidas del sistema.

En el caso de que todas las instituciones pudieran migrar de una figura a otra, el diagrama de transición quedaría descrito por el diagrama 2.1 que se presenta a continuación:

Diagrama 2.1. Transiciones posibles de las instituciones financieras

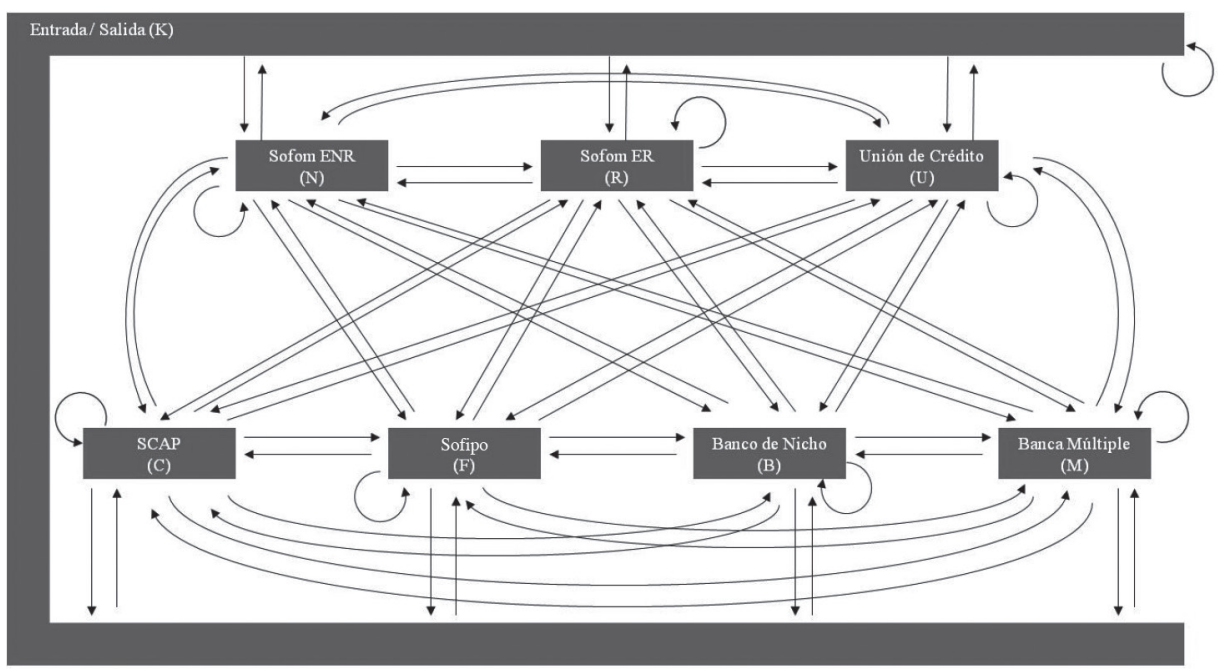

Fuente: Elaboración propia con base en datos de la CNBv y de la Condusef.

En la realidad muchas de las posibles transiciones que plantea el diagrama 2.1 no se han presentado. En la siguiente sección se especifican las transiciones que en efecto ocurrieron.

${ }^{17}$ Como es el caso de Unión de Crédito Unicredi, cuya autorización de operación fue revocada en 2011 y volvió a ingresar al sistema en 2013 bajo la misma figura (CNBV, 2007-2014d). 


\section{TRANSICIONES OCURRIDAS EN EL SECTOR FINANCIERO}

\section{III.1 Fuentes de información}

La información empleada para identificar a las instituciones financieras y las transiciones que éstas tuvieron en el periodo proviene principalmente de dos fuentes: 1) Libro Blanco Relativo a Autorizaciones, Fusiones y Revocaciones de Entidades Financieras (CNBV, 2006-2012), y 2) Boletines Estadísticos (CNBV, 2007-2014a-d).

En octubre de 2012 la CNBV (2006-2012) emitió un Libro Blanco Relativo a Autorizaciones, Fusiones y Revocaciones de Entidades Financieras. En él se detallan dichos movimientos suscitados en el periodo 2006-2012 para algunas instituciones financieras entre las que se encuentran las UC, SCAP, Sofipo, Instituciones de Banca Múltiple y Bancos de Nicho. Además, dichas instituciones reportan mensual o trimestralmente información de su desempeño a la CNBV, misma que se concentra y emite en los Boletines Estadísticos correspondientes (CNBV, 2007-2014a-d).

Las Sofom ER reportan a la CNBV su índice de capitalización, mismo que también es informado al público. Las listas del índice de capitalización (CNBV, 2007-2014e) también forman parte de la información fuente para identificar las transiciones en el periodo.

Las Sofom ENR deben cumplir con una regulación básica, principalmente centrada en la prevención de actividades de procedencia ilícita. Estas instituciones han sido más "reguladas" por el mercado (por ejemplo, por la Banca de Desarrollo y los fondeadores potenciales). En particular, la Comisión Nacional para la Protección y Defensa de los Usuarios de Servicios Financieros (Condusef, 2013, 2014) mantiene control del número de Sofom ENR que existen en el país y el detalle de cuáles de estas instituciones están realmente operando.

Se utilizó información disponible de la Condusef $(2012,2014)$ para mapear el sector, sin embargo, hay varios huecos en la información; por ejemplo, no se tiene información disponible de las Sofom ENR que dejaron de operar en cada año, o el nombre específico de las que estaban operando en un año determinado.

En algunos casos fue necesario recurrir a fuentes de información adicionales para mapear adecuadamente la transición. Tales fuentes incluyen: consultas al Diario Oficial de la Federación (2005-2014), análisis de Estados Financieros (2008-2013), evaluaciones de Entidades Calificadoras, y en algunos casos incluso la consulta directa a sitios de internet de las entidades.

En todos los casos se buscó la información al cierre de cada año (diciembre), esto fue particularmente complicado para 2014, puesto que la información disponible al momento de realizar la investigación corresponde a meses previos a 
diciembre. En tal caso se tomó la información disponible más reciente. Dichas transiciones se muestran a continuación.

\section{III.2 Transiciones ocurridas en el periodo 2007-2014}

Al inicio de 2007 había 609 instituciones financieras. Al cierre de 2014 se tenían 4,087. Lo que se traduce en un crecimiento promedio anual de 31.25 por ciento. En este apartado analizamos la (re)composición del sistema financiero mexicano a través de las transiciones ocurridas el periodo 2007-2014.

Nótese que, aunque en este aparado no se construyen Cadenas de Markov como tal, se sigue su lógica para construir cada una de las tablas. Las transiciones que ocurrieron durante el periodo están descritas en la tabla 3.1. La matriz de transición está en la tabla 3.2. Al construirlas se tomaron las siguientes consideraciones:

- $K j$ contempla las entradas al conjunto de las entidades descritas en el alcance, mientras que $K j$ representa las salidas para $j=[N, R, U, C, F, B, M]$.

- $K K=0$, puesto que si alguna institución entró y salió del mercado entre diciembre del año $n$ y diciembre del año $n+1$ (o viceversa), este caso quedó fuera de la información analizada.

- El otro valor de cero en la diagonal $(B B)$ se debe a que los primeros Bancos de Nicho se autorizaron hasta 2012 (CNBV, 2007-2014a, 2012c, 2006-2012).

- El movimiento UB y el BK corresponden a la misma institución (2012 UB y 2014 BK). El Banco Bicentenario era UC. En 2012 le dieron la autorización para convertirse en Banco de Nicho (B). Sin embargo, durante 2013-14 la CNBV decidió revocarle dicha autorización, por lo que se registró el movimiento BK.

- Se pude observar un número desproporcionalmente mayor de Sofom ENR que el resto de las figuras financieras $(K N) .{ }^{18}$

- Aunque no ha sido posible identificar el origen de las Sofom ENR, sí ha sido posible identificar el origen de las otras instituciones, por lo que sí se tiene la información de $j N$ y $N j$ para $j=[R, U, C, F, B, M]$.

- Tampoco se tiene información suficiente de las Sofom EnR que hayan salido del mercado, por lo que no hay información suficiente para construir $N K .{ }^{19}$ Por tal,

${ }^{18}$ Para el cierre de noviembre de 2014, la Condusef $(2012,2013,2014)$ tenía información de 3,727 Sofomes ENR registradas, mientras que para 2007 únicamente reportaba 340.

${ }^{19}$ La Condusef (2012) reporta que se han liquidado, revocado o fusionado 183 entidades, pero no describe en qué años se dieron estos movimientos, ni diferencia las fusiones de las salidas (liquidaciones y revocaciones). Sin embargo, sí se traza la transición $N K$ en el diagrama 3.1. Esta 
se asume que todos los cambios no identificados son entradas al sector $(K N)$. En este caso no se puede identificar la "doble transición" en el periodo 2007-2014.

Tabla 3.1. Transiciones ocurridas en el periodo 2007-2014

\begin{tabular}{|c|c|c|c|c|c|c|c|c|c|}
\hline & \multicolumn{8}{|c|}{2014} \\
\hline & & $\mathrm{N}$ & $R$ & $U$ & C & $\mathrm{F}$ & $B$ & $M$ & K \\
\hline \multirow{8}{*}{ 仓̊ㅁ } & $\mathrm{N}$ & 287 & 3 & 0 & 0 & 5 & 1 & 1 & 43 \\
\hline & $R$ & 2 & 12 & 0 & 0 & 0 & 0 & 0 & 5 \\
\hline & U & 0 & 0 & 90 & 0 & 0 & 1 & 0 & 50 \\
\hline & C & 0 & 0 & 0 & 55 & 4 & 0 & 0 & 1 \\
\hline & $\mathrm{F}$ & 0 & 0 & 0 & 0 & 19 & 0 & 0 & 0 \\
\hline & B & 0 & 0 & 0 & 0 & 0 & 0 & 0 & 1 \\
\hline & $\mathrm{M}$ & 0 & 0 & 0 & 0 & 0 & 0 & 35 & 5 \\
\hline & K & 3,438 & 12 & 13 & 80 & 22 & 3 & 5 & 0 \\
\hline
\end{tabular}

Fuente: Elaboración propia con datos de la CNBV y de la Condusef.

A continuación construimos la Cadena de Markov correspondiente. Para ello se sigue un método simple: cada probabilidad corresponde a la proporción de instituciones que cambiaron de un estado particular a otro estado durante el periodo considerado. De este modo, la Cadena de Markov para el periodo 2007-2014 queda de la siguiente manera:

Tabla 3.2. Matriz de transición 2007-2014

\begin{tabular}{|c|c|c|c|c|c|c|c|c|c|}
\hline & \multicolumn{8}{|c|}{2014} \\
\hline & & $N$ & $R$ & U & $C$ & $F$ & B & M & K \\
\hline \multirow{8}{*}{ ర్ } & $\mathrm{N}$ & 0.8441 & 0.0088 & 0.0000 & 0.0000 & 0.0147 & 0.0029 & 0.0029 & 0.1265 \\
\hline & $\mathrm{R}$ & 0.1053 & 0.6316 & 0.0000 & 0.0000 & 0.0000 & 0.0000 & 0.0000 & 0.2632 \\
\hline & U & 0.0000 & 0.0000 & 0.6383 & 0.0000 & 0.0000 & 0.0071 & 0.0000 & 0.3546 \\
\hline & C & 0.0000 & 0.0000 & 0.0000 & 0.9167 & 0.0667 & 0.0000 & 0.0000 & 0.0167 \\
\hline & $\mathrm{F}$ & 0.0000 & 0.0000 & 0.0000 & 0.0000 & 1.0000 & 0.0000 & 0.0000 & 0.0000 \\
\hline & B & 0.0000 & 0.0000 & 0.0000 & 0.0000 & 0.0000 & 0.0000 & 0.0000 & 1.0000 \\
\hline & $\mathrm{M}$ & 0.0000 & 0.0000 & 0.0000 & 0.0000 & 0.0000 & 0.0000 & 0.8750 & 0.1250 \\
\hline & $K$ & 0.9622 & 0.0034 & 0.0036 & 0.0224 & 0.0062 & 0.0008 & 0.0014 & 0.0000 \\
\hline
\end{tabular}

Fuente: Elaboración propia con datos de la CNBv y de la Condusef.

indicación de $N K$ también es viable y necesaria dado que de 2013 a 2014 el número de Sofom ENR registradas se redujo. 
El diagrama de transición que representa la información de la tabla 3.2 es el siguiente:

Diagrama 3.1. Transición de las instituciones financieras en el periodo 2007-2014

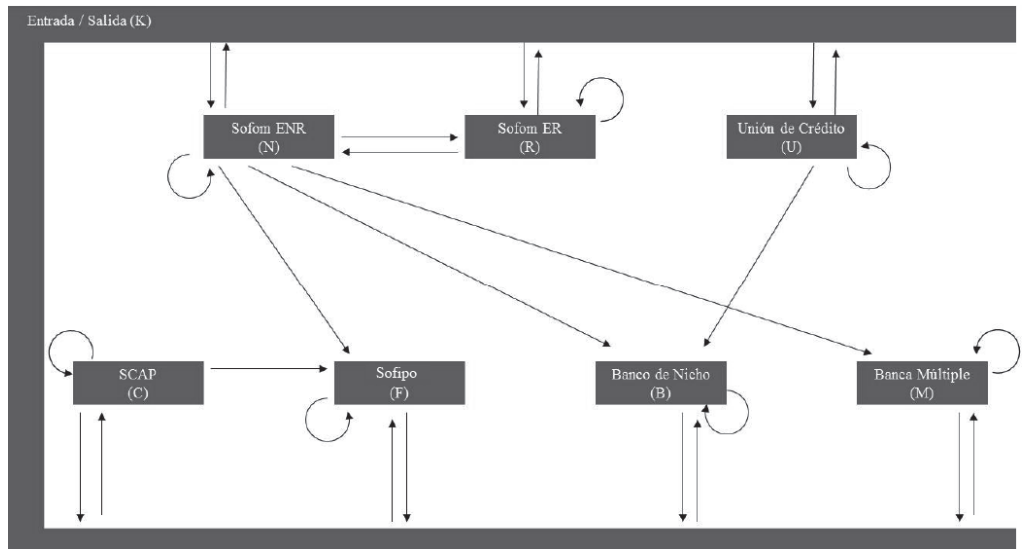

Fuente: Elaboración propia con datos de la CNBV y de la Condusef.

Partiendo de las tasas o probabilidades de transición contenidas en la tabla 3.2 y el diagrama 3.1, es inmediato darse cuenta que cualquier institución que busque entrar en el sector se constituirá con mayor probabilidad como Sofom ENR. Este hecho está vinculado, evidentemente, con la escasa regulación que existe para constituir este tipo de instituciones (SHCP, 2006) y los costos de entrada relativamente bajos.

\section{III.3 (Re)composición del sistema financiero mexicano}

La tabla 3.2 presenta las probabilidades de transición estimadas para el periodo 2007-2014. Los resultados más importantes que se derivan de ella son: 1) las Sofipo son las instituciones más estables al no mudarse a otra institución financiera y no registrar salida. No obstante, la probabilidad de que una institución entrante se constituya como Sofipo es muy baja. Existe de igual manera una probabilidad positiva de que algunas Sofom ENR y SCAP se conviertan en Sofipo; 2) las SCAP también son muy estables. Sólo pueden mudarse con baja probabilidad a Sofipo o salir del sistema. La probabilidad de que una institución entrante se constituya como SCAP es baja; 3) la Banca Múltiple resulta interesante debido a que la única transición que puede realizar consiste en salir del sistema. Además, la probabilidad de que una institución entrante se constituya como de Banca Múltiple es 
baja; 4) las Sofom ENR son las instituciones financieras que presentan mayor dinamismo. Hay una probabilidad alta de que las Sofom ENR no cambien de estado. No obstante, existe una probabilidad baja de que cambien a Sofipo, Banco de Nicho, Banca Múltiple o salir del sistema. Resulta muy destacable que existe una alta probabilidad de que una institución entrante se constituya como Sofom ENR. Lo que sugiere que el crecimiento del sector financiero mexicano implicará principalmente un crecimiento del número de las Sofom ENR. También es de hacer notar que algunas Sofom ER se muden a Sofom ENR; 5) las Sofom ER y Unión de Crédito resultaron ser las más inestables al tener las probabilidades más bajas en la diagonal. Dichas instituciones pueden salir del sistema con mayor probabilidad que las demás.

Además, se puede profundizar en diversos aspectos relevantes: la autorización de SCAP en el periodo (ver tabla 3.1) no debe entenderse como la entrada de "nuevos participantes en el sector", puesto que su origen proviene principalmente de las Cajas Populares, la mayoría de las cuales tienen décadas de haberse constituido. Sin embargo, el hecho de que estas entidades hayan sido autorizadas y operen en un entorno mejor regulado puede constituir una sólida base para futuros proyectos de desarrollo, dado que:

- Una SCAP autorizada puede ser (y es en algunos casos) un importante vehículo para que los programas gubernamentales (por ejemplo, implementados por Fira o Pronafim) lleguen a zonas rurales, población en situación de pobreza y pobreza extrema, productores del sector primario, otros sectores vulnerables, microempresarios y, en general, a la gran proporción de la población mexicana que no tiene acceso a los servicios financieros ofrecidos por la banca.

- Como el análisis lo muestra, aunque en el periodo 2007-2008 hubo algunas transiciones de la figura de SCAP a la Sofipo, éstas no han vuelto a ocurrir. Esto podría relacionarse con que: 1) las SCAP no se rigen por fines de lucro y 2) la publicación de las Disposiciones aplicables a las SCAP (CNBV, 2012a) reduce el posible interés de estas entidades por migrar a una figura diferente a la propia.

- Las SCAP generalmente ofrecen tasas de interés más competitivas y programas (aunque básicos) para fomentar la educación financiera en la población.

Por estos motivos es fundamental que se siga fortaleciendo a estas entidades y fomentando la incorporación de las que aún se encuentran en situación de prórroga condicionada.

La incorporación de los Bancos de Nicho pareció, al inicio (2009-2011), poco atractiva para el sector o no fue fomentada por las entidades reguladoras. Sin 
embargo, desde 2012 se incorporaron cinco entidades bajo esta figura. Como se observó en el diagrama 3.1, el origen de los Bancos de Nicho es diverso. Estas entidades eran previamente Sapi (PagaTodo), Sofol (Bankaool), IAP (Dondé), UC (Bicentenario), o Sofom ENR (Forjadores).

Ciertamente la incorporación de nuevas entidades podría promover la competencia en la banca. Sin embargo, valdría la pena analizar caso por caso qué otros beneficios podría traer la incorporación de estos bancos al sistema. Una posibilidad es que parezca atractivo para las Sofom ENR y que así busquen entrar en un marco regulado a un menor costo (por los menores requerimientos de capital); sin embargo, de los cinco Bancos de Nicho, únicamente Forjadores proviene del sector de las Sofom ENR. Por otro lado, la rápida salida de Banco Bicentenario, un año después de haber sido autorizado para operar como Institución de Banca Múltiple, puede generar incertidumbre y desconfianza en este tipo de instituciones.

Las Sofipo aparecen como una figura atractiva y estable para el sector. En la tabla 3.1 se muestra que algunas Sofom ENR, SCAP y otras entidades han buscado su incorporación a esta figura. Por otro lado, estas entidades generalmente, atienden a sectores similares a los de las SCAP; ofrecen servicios financieros más competitivos que las Sofom ENR y se desempeñan en un marco regulado. Por lo que pueden ser un buen vehículo para la aplicación de programas de apoyo gubernamental. Al igual que con los Bancos de Nicho, la reciente salida de Ficrea del sector puede ser un detonante de desconfianza en estas instituciones.

Durante el periodo de análisis también hubo importantes salidas del sector, principalmente para el caso de las UC. La revocación de autorización para la operación de estas entidades por parte de la CNBV se puede vincular con irregularidades identificadas. En este sentido, aunque la salida de estas entidades podría no impactar directamente a la inclusión financiera, sí puede incrementar la confiabilidad en el sistema financiero. Esto también es importante porque gran parte de estas entidades se desempeñan en el sector primario (agricultura, ganadería, pesca, entre otros) y en zonas rurales y semiurbanas, lo que podría vincular a las UC con la población objetivo de la inclusión financiera.

\section{III.4 El sector financiero y los servicios financieros relevantes}

Un estudio del Center for Financial Inclusion (2009) identifica una variedad de servicios financieros de mayor relevancia para la población de bajos ingresos. Estos servicios se contrastaron con las operaciones que pueden realizar las instituciones financieras analizadas. Los resultados se presentan en la tabla 3.3: 
152 ECONOMÍA TEORÍA Y PRÁCTICA • Nueva Época, número 47, julio-diciembre 2017

Tabla 3.3. Servicios financieros relevantes para la población de bajos ingresos que pueden ser ofrecidos por las instituciones analizadas

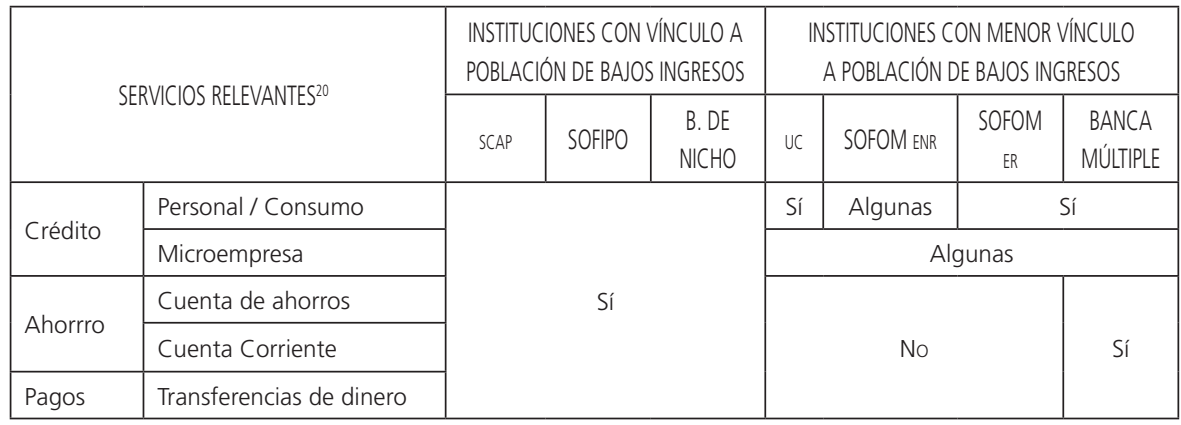

Fuente: Elaboración propia contrastando los servicios relevantes para la población de bajos recursos con las operaciones permisibles para cada tipo de institución (Center for Financial Inclusion, 2009; CNBV, 2012a, 2012b, 2013a, 2013b; SHCP, 2006).

En la tabla 3.3 se contemplan las SCAP, las Sofipo y los Bancos de Nicho como mayormente vinculados a la población de bajos ingresos al ofrecerle los servicios necesarios que requiere. Como se describió, las primeras dos constituyen las Entidades de Ahorro y Crédito Popular y están fuertemente vinculadas con la población objetivo para la inclusión financiera. Los Bancos de Nicho se incluyen en este grupo puesto que al menos tres de ellos operan con la población objetivo (Forjadores, Bankaool y Dondé).

El hecho de que este grupo de instituciones tengan dentro de su abanico de operaciones permisibles los servicios financieros que se consideran básicos en materia de inclusión financiera integral, refuerza la necesidad de continuar fomen-

${ }^{20}$ De acuerdo con el Center for Financial Inclusion (2009) éstos son los servicios "fundamentales" para la inclusión financiera integral. Una variedad completa de servicios necesarios para la población de bajos ingresos incluye otros productos de:

- Crédito: tarjeta de crédito, educación, hipotecario, remodelación de vivienda, activo fijo de negocio y arrendamiento;

- Ahorro: certificado de depósito, pensión, ahorro de jóvenes y programas de ahorro;

- Seguros: de vida, salud, propiedad, discapacidad y cultivo. y

- Pagos: transferencias internacionales, pago de recibos, transferencias y beneficios del gobierno, nómina y pagos electrónicos.

Aunque el marco regulatorio establece las operaciones permitidas para las diferentes entidades, sería necesario un análisis más específico para evaluar si las instituciones del sistema están ofreciendo efectivamente estos servicios al público. 
tando la incorporación y fortalecimiento de este tipo de instituciones al sistema financiero.

El vínculo de las otras instituciones (Banca Múltiple, Sofom y UC) con la población de bajos ingresos es menos claro. ${ }^{21}$ Más aún, las Sofom y las UC no están autorizadas para captar ahorro y un número no determinado de estas instituciones tampoco otorgan microcréditos.

En este punto se puede contrastar la información de la tabla 3.3 sobre servicios financieros relevantes con las transiciones registradas en la tabla 3.2. Particularmente con la enorme incorporación de Sofom ENR. Debido a su escasa regulación y a la poca vinculación con la población de bajos ingresos, la incorporación de estas instituciones al sistema implica importantes riesgos para la población mexicana como son: sobreendeudamiento, mayor oferta de servicios poco competitivos, baja confiabilidad en el sistema, entre otros.

De lo anterior se considera que las actividades para el sector financiero deberían incorporar un mayor control sobre las Sofom ENR. Algunas alternativas para ello pueden contemplar: incrementar las restricciones para incorporar estas entidades, fomentar su cambio a figuras reguladas, o ajustar su marco regulatorio para convertirlas en entidades más competitivas y confiables. A este respecto, alguna Sofom ENR han buscado migrar a figuras reguladas, pero la incorporación de nuevas entidades como Sofom ENR es mucho más acelerada.

Adicionalmente, existen otros elementos relevantes de las políticas y programas desarrollados para la inclusión financiera integral como son: la incorporación de corresponsales bancarios, la prestación de servicios financieros a través de comisionistas móviles y el régimen de cuentas simplificadas, entre otras. Por quedar fuera del alcance de este artículo, tales políticas y programas no se consideran.

\section{El FUTURO DE LAS INSTITUCIONES FINANCIERAS}

En esta sección realizamos un pronóstico de la composición de las instituciones financieras en México al cierre del año 2015. Para ello, nos auxiliamos de la Cadena de Markov del año 2013-2014.

\footnotetext{
${ }^{21} \mathrm{Sin}$ duda, hay algunas de estas instituciones que ofrecen sus servicios a la población de bajos ingresos. Ejemplos de ello son Banco Compartamos; Grameen Carso, Sofom ER; Apoyo Integral México, Sofom ENR; algunas UC enfocadas a la operación en el sector primario y en zonas rurales, así como las instituciones inscritas a los programas de apoyo de Financiera Rural. Sin embargo, para cada uno de estos ejemplos, en este segundo grupo de instituciones hay múltiples contraejemplos de entidades que están enfocadas a la población de ingresos medios y altos.
} 
Para validar, al menos en parte, la metodología que estamos implementando en este capítulo, primero realizaremos la estimación para el cierre del año 2014. De este modo podemos verificar la precisión de esta estimación al compararla con la composición que en efecto se tuvo al cierre del año 2014. Posteriormente repetiremos el procedimiento para estimar la composición de las instituciones financieras al cierre del año 2015.

\section{IV.1 Transiciones ocurridas en el periodo 2012-2013}

La tabla 4.1 escribe las transiciones ocurridas en el periodo 2012-2013. Al construirla se tomaron las siguientes consideraciones:

- La Sofol Consupago y tres OAC se constituyen como Sofom ER. Tres entidades instituciones dejan de operar bajo esta figura, dos de las cuales se relacionan con las negociaciones de fusión entre Ixe y Banorte (CNBV, 2007-2014e; Diario Oficial de la Federación, 2005-2014; Estados Financieros, 2008-2013).

- Santander adquirió el capital de ING Hipotecaria Sofom ENR para conformar Santander Vivienda, Sofom ER (CNBv, 2007-2014e; Diario Oficial de la Federación, 2005-2014).

- Una Sofom ENR (Crediclub) y una S.A. de C.V. (smb Rural) fueron autorizadas para operar como Sofipo (CNBV, 2007-2014c; Diario Oficial de la Federación, 2005-2014).

- Ucredi, cuya autorización fue revocada en 2011, vuelve a ser autorizada como UC. Se revoca la autorización a dos UC (CNBV, 2007-2014d).

- Se revocó la autorización a 16 UC (CNBV, 2007-2014d).

- Como se describió previamente, ante el requerimiento de la CNBV para la regulación de las Cajas Populares, más instituciones presentaron su solicitud de autorización. En total fueron 63 las SCAP autorizadas en el año (CNBV, 20072014b, 2013c-d).

- Financiera Sustentable de México y Ku-Bo Sofom enr son autorizadas como Sofipos (CNBV, 2007-2014c; Diario Oficial de la Federación, 2005-2014).

- Fundación Rafael Dondé, IAP recibe su autorización para operar como Banca Múltiple, dada la información de su capital social se incorpora como Banco de Nicho (CNBV, 2007-2014a; Diario Oficial de la Federación, 2005-2014).

- La Sapi PagaTodo también se autoriza como Banca Múltiple; esta entidad no reportó información de su capital al cierre de 2013, sin embargo, dados las operaciones que pretende realizar (medios de pago) y el marco regulatorio 
establecido por la CNBV, su figura corresponde a Banco de Nicho (CNBV, 20072014a; Diario Oficial de la Federación, 2005-2014).

- Hipotecaria Casa Mexicana Sofom ENR es autorizada para operar como banco con el nombre de Banco Inmobiliario Mexicano. Su capital social excede los 90 millones de UDIS por lo que se considera dentro del grupo de Instituciones de Banca Múltiple (CNBV, 2007-2014a; Diario Oficial de la Federación, 2005-2014).

- En el norte de México, Banorte y otras S.A de C.V. aportan capital para la creación de una Institución de Banca Múltiple denominada Bancrea. Al igual que PagaTodo, a la fecha del análisis Bancrea no reportó información financiera en el Boletín Estadístico. Sin embargo, dada la información financiera reportada en el primer Boletín Estadístico de 2014, se observa que el capital social de Bancrea excede los 90 millones de UDIS, por lo que su entrada es considerada como Institución de Banca Múltiple (CNBV, 2007-2014a; Diario Oficial de la Federación, 2005-2014).

- Ante la fusión de Ixe y Banorte, Ixe deja de reportar información a la CNBV como Institución de Banca Múltiple (CNBV, 2007-2014a; Diario Oficial de la Federación, 2005-2014; Estados Financieros (2008-2013)).

- En 2013 la CNBV (2007-2014a) revocó la autorización de ING para operar como Institución de Banca Múltiple.

- No se cuenta con información detallada del cierre 2013 respecto a las Sofomes ENR, por lo que se hacen las mismas consideraciones descritas previamente en el documento (Condusef, 2013, 2014).

Tabla 4.1. Transiciones ocurridas en el periodo 2012-2013

\begin{tabular}{|l|l|}
\hline \multicolumn{2}{|c|}{2012} \\
\hline \multicolumn{1}{|c|}{$\#$} & \multicolumn{1}{c|}{$\%$} \\
\hline 2,755 & $90.2 \%$ \\
\hline 25 & $0.8 \%$ \\
\hline 119 & $3.9 \%$ \\
\hline 69 & $2.3 \%$ \\
\hline 43 & $1.4 \%$ \\
\hline 3 & $0.1 \%$ \\
\hline 42 & $1.4 \%$ \\
\hline
\end{tabular}

\begin{tabular}{|c|c|c|c|c|c|c|c|c|c|}
\hline \multicolumn{2}{|c|}{} & \multicolumn{10}{|c|}{2013} \\
\hline \multicolumn{1}{|c|}{} & $\mathrm{N}$ & $\mathrm{R}$ & $\mathrm{U}$ & $\mathrm{C}$ & $\mathrm{F}$ & $\mathrm{B}$ & $\mathrm{M}$ & $\mathrm{K}$ \\
\hline & $\mathrm{N}$ & 2,751 & 1 & 0 & 0 & 2 & 0 & 1 & 0 \\
\hline & $\mathrm{R}$ & 0 & 22 & 0 & 0 & 0 & 0 & 0 & 3 \\
\hline & $\mathrm{U}$ & 0 & 0 & 103 & 0 & 0 & 0 & 0 & 16 \\
\cline { 2 - 11 }$\approx$ & $\mathrm{C}$ & 0 & 0 & 0 & 68 & 0 & 0 & 0 & 1 \\
\cline { 2 - 11 } & $\mathrm{F}$ & 0 & 0 & 0 & 0 & 43 & 0 & 0 & 0 \\
\hline & $\mathrm{B}$ & 0 & 0 & 0 & 0 & 0 & 3 & 0 & 0 \\
\hline & $\mathrm{M}$ & 0 & 0 & 0 & 0 & 0 & 0 & 40 & 2 \\
\hline & $\mathrm{K}$ & 1,021 & 4 & 1 & 63 & 2 & 2 & 1 & 0 \\
\hline
\end{tabular}

\begin{tabular}{|c|c|}
\hline \multicolumn{2}{|c|}{2013} \\
\hline$\#$ & $\%$ \\
\hline 3,772 & $91.4 \%$ \\
\hline 27 & $0.7 \%$ \\
\hline 104 & $2.5 \%$ \\
\hline 131 & $3.2 \%$ \\
\hline 47 & $1.1 \%$ \\
\hline 5 & $0.1 \%$ \\
\hline 42 & $1.0 \%$ \\
\hline
\end{tabular}

Fuente: Elaboración propia con datos de la CNBV y de la Condusef. 
156 ECONOMÍA TEORÍA Y PRÁCTICA • Nueva Época, número 47, julio-diciembre 2017

A continuación se presenta la Cadena de Markov para el periodo 2012-2013:

Tabla 4.2. Matriz de transición 2012-2013

\begin{tabular}{|c|c|c|c|c|c|c|c|c|c|}
\hline & \multicolumn{8}{|c|}{2013} \\
\hline & & $\mathrm{N}$ & $\mathrm{R}$ & U & C & $\mathrm{F}$ & B & $M$ & K \\
\hline \multirow{8}{*}{$\stackrel{\sim}{\check{2}}$} & $\mathrm{~N}$ & 0.9985 & 0.0004 & 0 & 0 & 0.0007 & 0 & 0.0004 & 0 \\
\hline & $\mathrm{R}$ & 0 & 0.88 & 0 & 0 & 0 & 0 & 0 & 0.12 \\
\hline & $U$ & 0 & 0 & 0.8655 & 0 & 0 & 0 & 0 & 0.1345 \\
\hline & $C$ & 0 & 0 & 0 & 0.9855 & 0 & 0 & 0 & 0.0145 \\
\hline & $\mathrm{F}$ & 0 & 0 & 0 & 0 & 1 & 0 & 0 & 0 \\
\hline & $B$ & 0 & 0 & 0 & 0 & 0 & 1 & 0 & 0 \\
\hline & $M$ & 0 & 0 & 0 & 0 & 0 & 0 & 0.9524 & 0.0476 \\
\hline & K & 0.9333 & 0.0037 & 0.0009 & 0.0576 & 0.0018 & 0.0018 & 0.0009 & 0 \\
\hline
\end{tabular}

Fuente: Elaboración propia con datos de la CNBv y de la Condusef.

IV.2 Estimaciones realizadas para el cierre de 2014

Procedemos a estimar la composición de instituciones financieras al cierre de 2014 utilizando la información del periodo previo (2012-2013). Para ello se tomaron las siguientes consideraciones:

- La matriz (4.2) se ajustó al 52 por ciento en las Sofom ENR debido a que la Condusef $(2012,2013,2014)$ reporta que 48 por ciento de las Sofom ENR no están operando. Por tal motivo, la matriz que utilizamos para realizar las estimaciones es la descrita en la tabla A1 del apéndice.

- Se tomó la información de 2013 como estado inicial .

- Para reflejar el incremento de instituciones, se consideraron tres escenarios:

1. El primer escenario supone que no entran nuevas instituciones, sólo hay transiciones de las existentes;

2. Un escenario de crecimiento moderado, considerando un incremento del 6 por ciento en el número de instituciones; ${ }^{22}$

${ }^{22}$ Crecimiento similar al observado en el periodo 2011-2012. 
3. Un escenario suponiendo un incremento del 30 por ciento en el número de instituciones. $^{23}$

- El número total de instituciones registradas a 2013 se multiplicó por los incrementos de los tres escenarios y se aplicó a la mezcla estimada por el estado de la matriz A1.

Los resultados de dicha estimación se muestran en el gráfico 4.1:

Gráfica 4.1. Estimación 2014. Matriz de transición 2012-213. Estado inicial 2013. Estado estimado: $\mathbf{S 1}$

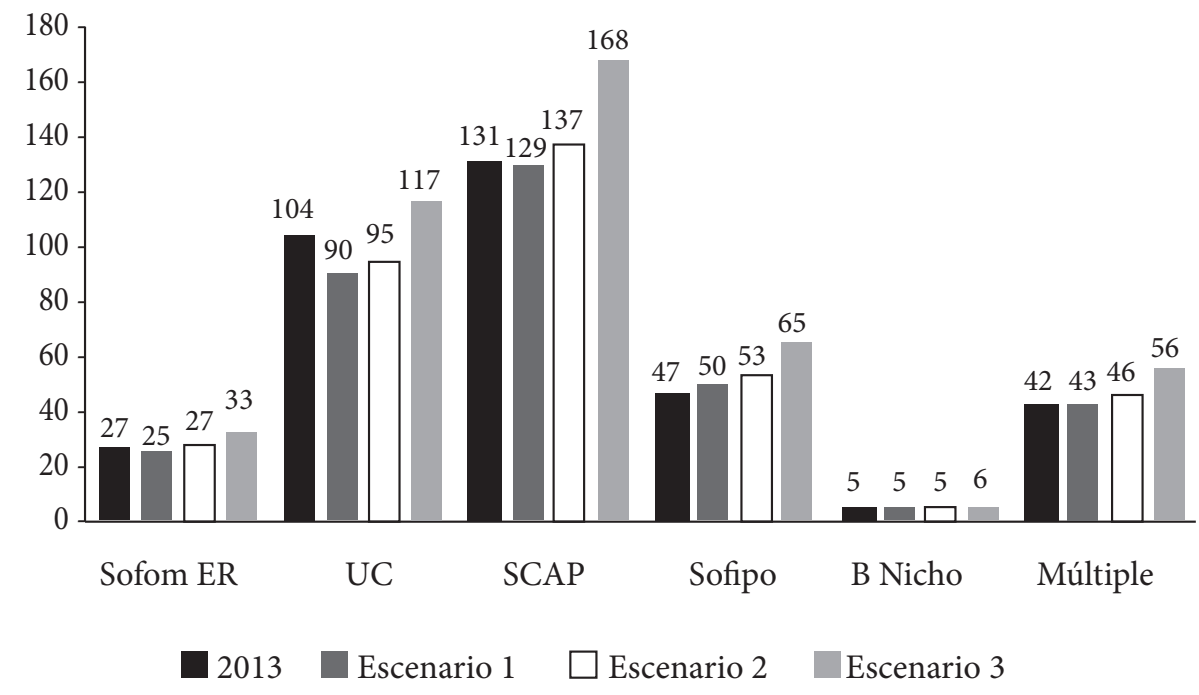

Fuente: Estimación propia a partir de datos de la CNBv y de la Condusef.

Observando la información del gráfico 4.1 y la matriz A1, la estimación para 2014 muestra que los resultados dependen del porcentaje en que aumentan las instituciones financieras. Detalladamente tenemos lo siguiente:

- Las Sofom ER podrían haberse reducido (de 27) a 25 (Escenario 1). La probabilidad de incorporación de nuevas instituciones como Sofom ER $\left(\mathrm{p}_{K R}=0.0066\right)$.

- Las SCAP (131) podrían haber disminuido a 129 (Escenario 1) o aumentado a 137 (Escenario 2). El Escenario 3 estima que en 2014 se autorizarían 37 entidades más. La probabilidad de ingreso al sistema bajo esta figura es elevada

${ }^{23}$ Crecimiento similar al incremento promedio observado en el periodo 2007-2013. 
$\left(\mathrm{p}_{K C}=0.1043\right)$, lo cual seguramente sería por la autorización de Cajas Populares ya existentes que entregasen su solicitud para ser autorizadas.

- Se estima un incremento en las Sofipo (47). Estas podían aumentar a 53 (Escenario 2). Incluso en el Escenario 1, que supone que no hay incorporación de nuevas instituciones, la estimación sugiere la transición de al menos una institución a esta figura. El incremento en el número de Sofipo podía ser por la entrada de nuevas instituciones $\mathrm{o}\left(\mathrm{p}_{K F}=0.0033\right)$ a través de la autorización de Sofomes ENR $\left(\mathrm{p}_{N F}=0.0014\right)$.

- Sólo en el Escenario 3 se estima que un Banco de Nicho se incorporase al mercado. Esta autorización se daría con mayor probabilidad para una institución "entrante" como una S.A. de C.V., Sapi o una IAP $\left(\mathrm{p}_{K B}=0.0033\right)$.

- El Escenario 2 estima la incorporación de cuatro Instituciones de Banca Múltiple en 2014. La estimación sugiere que estos bancos podían conformarse a partir de la autorización de Sofom ENR $\left(\mathrm{p}_{N M}=0.0007\right)$ o por la incorporación de nuevas instituciones, por ejemplo, S.A. de C.V ( $\left.\mathrm{p}_{K M}=0.0017\right)$. Dado que en 2013 dos Instituciones de Banca Múltiple salieron del sistema por fusión (Ixe) o revocación (ING), la estimación de salida de estas instituciones para 2014 es elevada $\left(\mathrm{p}_{M K}=0.0476\right)$.

- Sólo el Escenario 3 estima incremento en el número de UC. Este resultado es interesante, puesto que se ha visto una reducción constante de estas entidades en los últimos años.

- Las Sofipo y los Bancos de Nicho constituyen estados absorbentes de la cadena $\left(\mathrm{p}_{F F}=\mathrm{p}_{B B}=1\right)$, por lo que la estimación sugiere que ninguna de estas instituciones sale del sistema financiero ni busca un cambio de figura.

- El impacto más importante (en cuanto al número de instituciones) está evidentemente en las Sofom ENR. Incluso aplicando el ajuste del 52 por ciento, en 2014 podrían haberse incorporado entre 110 (Escenario 2) y 579 entidades (Escenario 3) bajo esta figura. La estimación sugiere que el incremento en este tipo de entidades sería por la entrada de nuevos participantes $\left(P_{K N}=0.8791\right){ }^{24}$

En el siguiente apartado comparamos los resultados estimados con las transiciones que en efecto ocurrieron al cierre de 2014.

\footnotetext{
${ }^{24}$ Dado que las cifras de las Sofom ENR son mucho mayores que las de las demás entidades, no se incluyó su información en el gráfico 4.1.
} 
IV.3 Estimaciones y observaciones para el cierre de 2014

La siguiente tabla muestra el comparativo de las estimaciones realizadas en la sección anterior y la composición de instituciones financieras observada al cierre de 2014:

Tabla 4.3. Datos estimados y observados al cierre de 2014

\begin{tabular}{|c|c|c|c|c|}
\hline \multirow{2}{*}{$\begin{array}{c}\text { TIPO DE INSTITU- } \\
\text { CIÓN }\end{array}$} & VALORES OBSERVADOS AL CIERRE DE & \multicolumn{3}{|c|}{ ESTIMACIONES A PARTIR DE LA MATRIZ 2012 - 2013 } \\
\cline { 3 - 5 } & 2014 & ESCENARIO 1 & ESCENARI0 2 & ESCENARI0 3 \\
\hline $\mathrm{N}$ & 1,938 & 1,955 & 2,072 & 2,541 \\
\hline $\mathrm{R}$ & 27 & 25 & 27 & 33 \\
\hline $\mathrm{U}$ & 103 & 90 & 95 & 117 \\
\hline $\mathrm{C}$ & 135 & 129 & 137 & 168 \\
\hline $\mathrm{F}$ & 49 & 50 & 53 & 65 \\
\hline $\mathrm{B}$ & 4 & 5 & 5 & 6 \\
\hline $\mathrm{M}$ & 41 & 43 & 46 & 56 \\
\hline
\end{tabular}

Fuente: Estimación propia a partir de datos de la CNBV y de la Condusef.

La tabla anterior muestra que los escenarios 1 y 2 fueron los que dieron resultados más similares a las transiciones ocurridas durante 2014.

Por un lado, en el Escenario 1 (escenario en el que no habría incremento de instituciones, únicamente transiciones), se observa que el estado $S_{I}$ presenta una estimación más cercana a los valores observados para las Sofipo, los Bancos de Nicho y las Instituciones de Banca Múltiple.

Por otro lado, en el Escenario 2 (con un incremento moderado del 6 por ciento en el número de Instituciones), la estimación es más certera para las Sofom ER, UC Y SCAP.

\section{IV.4 Estimaciones realizadas para el cierre de 2015}

Procedemos a estimar de la mezcla de instituciones financieras al cierre de 2015 utilizando ahora la información del periodo previo (2013-2014). Para ello se considera lo siguiente:

- Se usó la matriz 2013-2014, considerando el ajuste ahora del 57 por ciento en las Sofom ENR. Esto dado que, según la información de la Condusef $(2013,2014)$ al cierre de 2014 , es ahora el 43 por ciento de este tipo de instituciones el que no está operando. 
- Se consideró también la salida de Ficrea del mercado de las Sofipo. Institución que fue intervenida por la CNBV por presentar irregularidades. Cabe destacar que este es el primer caso que se observa de la salida de una Sofipo (FK) en el periodo de análisis.

- Incluyendo estas dos consideraciones la matriz de transición ajustada es: ${ }^{25}$

Tabla 4.4. Matriz de transición 2013-2014 ajustada

\begin{tabular}{|c|c|c|c|c|c|c|c|c|c|}
\hline & & \multicolumn{10}{|c|}{2014} & & \\
\hline & & $\mathrm{N}$ & $\mathrm{R}$ & $\mathrm{U}$ & $\mathrm{C}$ & $\mathrm{F}$ & $\mathrm{B}$ & $\mathrm{M}$ & $\mathrm{K}$ \\
\hline \multirow{5}{*}{$\underset{\mathrm{m}}{\mathrm{m}}$} & $\mathrm{N}$ & 0.9874 & 0.0005 & 0 & 0 & 0.0005 & 0 & 0 & 0.0116 \\
\cline { 2 - 10 } & $\mathrm{R}$ & 0 & 0.9630 & 0 & 0 & 0 & 0 & 0 & 0.0370 \\
\cline { 2 - 10 } & $\mathrm{U}$ & 0 & 0 & 0.9904 & 0 & 0 & 0 & 0 & 0.0096 \\
\cline { 2 - 10 } & $\mathrm{C}$ & 0 & 0 & 0 & 1 & 0 & 0 & 0 & 0 \\
\cline { 2 - 10 } & $\mathrm{F}$ & 0 & 0 & 0 & 0 & 0.9787 & 0 & 0 & 0.0213 \\
\cline { 2 - 10 } & $\mathrm{B}$ & 0 & 0 & 0 & 0 & 0 & 0.8000 & 0 & 0.2000 \\
\cline { 2 - 10 } & $\mathrm{M}$ & 0 & 0 & 0 & 0 & 0 & 0 & 0.9762 & 0.0238 \\
\cline { 2 - 10 } & $\mathrm{K}$ & 0 & 0 & 0 & 0.6667 & 0.3333 & 0 & 0 & 0 \\
\hline
\end{tabular}

Fuente: Elaboración propia con datos de la CNBV y de la Condusef.

- Se tomó entonces la información del cierre 2014 como estado inicial (también con el ajuste al 57 por ciento sobre las Sofom ENR). Dados los resultados del apartado anterior, se consideran sólo dos escenarios para predecir el incremento de instituciones:

1.- El primero que no considera nuevas entradas.

2.- El segundo de crecimiento moderado del 6 por ciento, igual que el escenario 2 de la sección 4.2 .

Los resultados de estas estimaciones se muestran en la tabla 4.5

Dadas las salidas presentadas en 2014 en general, el Escenario 1 (sin crecimiento) también sugiere salidas de UC, Bancos de Nicho e Instituciones de Banca Múltiple, pero principalmente de algunas Sofom ENR.

El Escenario 2 por su parte sugiere incrementos en todas las figuras de instituciones financieras excepto en la Banca de Nicho.

${ }^{25}$ La matriz de transición sin ajustar se presenta en el apéndice en la tabla A2. 
De la tabla 4.5 se observa que, independientemente de las salidas del sector, seguirá predominando en gran medida la figura de las Sofom ENR. Hecho que, como se dijo antes, podría implicar importantes riesgos a la población mexicana, principalmente a la de bajos recursos.

Tabla 4.5. Estimaciones para el cierre 2015

\begin{tabular}{|l|c|c|c|}
\hline \multirow{2}{*}{2014,} & \multicolumn{2}{|c|}{ ESTIMACIÓN 2015 } \\
\cline { 2 - 4 } & VALORES OBSERVADOS & ESCENARI0 1 & ESCENARI02 \\
\hline Sofom ENR* & 2,124 & 2,097 & 2,223 \\
\hline Sofom ER & 27 & 27 & 29 \\
\hline UC & 103 & 102 & 108 \\
\hline SCAP & 135 & 135 & 143 \\
\hline Sofipo & 49 & 49 & 52 \\
\hline B Nicho & 4 & 3 & 3 \\
\hline B Múltiple & 41 & 40 & 42 \\
\hline
\end{tabular}

Fuente: Elaboración propia con datos de la CNBV y de la Condusef.

* Nótese que este valor es diferente al correspondiente en la tabla 4.3. Ello se debe a la diferencia en el ajuste para las Sofomes ENR. Es decir, en el ejercicio de la sección 4.3 se consideró toda vía un ajuste del 52 por ciento, mientras que para este ejercicio el ajuste es del 57 por ciento.

\section{Conclusiones}

El presente trabajo analiza la (re)composición del sistema financiero mexicano utilizando Cadenas de Markov. En el periodo 2007-2014 se registró un enorme incremento de instituciones financieras registrando un crecimiento promedio anual de 31.25 por ciento. Del total de instituciones financieras nuevas, hubo un incremento importante de instituciones que son mayormente vinculadas con la población de bajos ingresos (SCAP y Sofipo). No obstante, existe una alta probabilidad de que una institución entrante se constituya como Sofom ENR. Estas entidades ofrecen pocos de los servicios financieros relevantes para la población de bajos ingresos. El análisis muestra que las Sofom ENR son las instituciones financieras que presentan mayor dinamismo al capturar la mayoría de entradas al sistema. Si bien, existe una probabilidad alta de que las Sofom ENR no cambien de estado, existe una probabilidad baja de que cambien a Sofipo, Banco de Nicho, Banca Múltiple o salir del sistema. 
La creciente tendencia de incorporación de Sofom ENR, en un marco no regulado, podría implicar algunos riesgos para la población mexicana.

El presente análisis sugiere que se deben contemplar algunas medidas relacionadas a las Sofom ENR como pueden ser: incrementar las restricciones para incorporar estas entidades, fomentar su cambio a figuras reguladas o ajustar su marco regulatorio para convertirlas en entidades más competitivas y confiables.

El uso de Cadenas de Markov para describir y analizar las transiciones de las instituciones financieras presenta algunas limitantes. No podemos garantizar que las probabilidades de transición sean estacionarias debido a que dichas probabilidades de transición se calculan de manera frecuencial. Lo que da lugar a que las Cadenas de Markov no necesariamente sean regulares y, como consecuencia, no se puedan utilizar para hacer predicciones que involucren varios años a futuro. Tampoco se puede encontrar el estado estable. En la medida en que se cuente con mejores fuentes de datos se podrán construir Cadenas de Markov con probabilidades de transición estacionarias.

Es de destacar que el presente artículo analiza la dinámica de la oferta de servicios financieros sin considerar el precio de dichos servicios. En un análisis más exhaustivo que involucre la competencia entre servicios financieros se debe incluir la evolución de sus precios, así como de los rendimientos asociados al ahorro/depósito de recursos, al menos de algunas de estas instituciones. Adicionalmente, los resultados del presente análisis dan lugar a nuevas interrogantes entre las que destacan: ¿Cómo impacta la (re)composición observada en el sistema financiero en la disponibilidad de mejores productos?; ¿En qué zonas del país y sectores de la población ha impactado esta (re)composición del sistema financiero?; ¿Existe alguna relación entre la (re)composición del sistema financiero, la educación financiera y la protección al consumidor?, y ¿Qué figura del sistema financiero ha resultado la más efectiva para fomentar la inclusión financiera?

\section{REFERENCIAS BIBLIOGRÁFICAS}

Avalos, Marcos y Hernández, Fausto (2006), Competencia bancaria en México, Serie Estudios y Perspectivas 62, México, Cepal, Publicación de Naciones Unidas.

Castellanos, Sara; Gustavo A. Del Angel y Garza-García, Jesus G. (2016), Competition and Efficiency in the Mexican Banking Industry, Theory and Empirical Evidence, Nueva York, Palgrave MacMillan. 
Center for Financial Inclusion (2009), Perspectivas para México de Inclusión Financiera Integral.

Comisión Nacional Bancaria y de Valores, (CNBV) (2006-2012), Libro Blanco Relativo a Autorizaciones, Fusiones y Revocaciones de Entidades Financieras, México. (2007-2014a), Boletín Estadístico de Banca Múltiple, México, [en línea], disponible en: http://portafoliodeinformacion.cnbv.gob.mx/bm1/Paginas/boletines.aspx. [Consultado el 30 de diciembre de 2014]. (2007-2014b), Boletín Estadístico: SCAP, México, [en línea], disponible en: http://portafoliodeinformacion.cnbv.gob.mx/eacp1/Paginas/boletines.aspx. [Consultado el 30 de diciembre de 2014]. (2007-2014c), Boletín Estadístico: Sofipo, México, [en línea], disponible en: http://portafoliodeinformacion.cnbv.gob.mx/eacp1/Paginas/boletines.aspx. [Consultado el 30 de diciembre de 2014]. (2007-2014d), Boletín Estadistico: Unión de Crédito, México, [en línea], disponible en: http://portafoliodeinformacion.cnbv.gob.mx/oaacs1/Paginas/boletines_uc.aspx. [Consultado el 30 de diciembre de 2014]. (2007-2014e), Índices de Capitalización de las Sofom ER, México, [en línea], disponible en: http://portafoliodeinformacion.cnbv.gob.mx/sofoles/Paginas/sm_alertas.aspx. [Consultado el 30 de diciembre de 2014]. (2012a), Disposiciones de Carácter General Aplicables a las Actividades de las Sociedades Cooperativas de Ahorro y Préstamo (SCAP), México.

(2012b), Disposiciones de Carácter General Aplicables a las Entidades de Ahorro y Crédito Popular y Organismos de Integración a que se refiere la Ley de Ahorro y Crédito Popular (Sofipo), México. (2012c), Libro Blanco de Inclusión Financiera, México. (2013a), Disposiciones de Carácter General Aplicables a las Instituciones de Crédito (Circular Única de Bancos-CUB), México.

(2013b), Disposiciones de Carácter General Aplicables a las Organizaciones Auxiliares del Crédito, Casas de Cambio, Uniones de Crédito, Sociedades Financieras de Objeto Limitado y Sociedades Financieras de Objeto Múltiple Reguladas (Cuife), México. (2013c) Informe Anual. México. (2013d), Sociedades Autorizadas para Operar al Amparo de la LRASCAP, México. 
Comisión Nacional para la Protección y Defensa de los Usuarios del Servicio Financiero, (Condusef) (2012), La importancia de las Sofom ENR para la Condusef, México, [en línea], disponible en: http://www.condusef.gob. $\mathrm{mx} / \mathrm{pdf}$-s/Comunicados/2012/com47_importancia-sofom.pdf. [Consultado el 30 de diciembre de 2014].

(2013, 2014), Estatus de Operación de las Sofom ENR. Listado de Sofomes ENR, México, [en línea], disponible en: http://www.condusef.gob.mx/ index.php/estatus-de-operacion-de-las-sofom-enr. [Consultado el 30 de diciembre de 2014].

Congreso de los Estados Unidos Mexicanos (2010), Ley de Instituciones de Crédito, México.

Di Giannatale, Sonia; López, Patricia y José Roa, María (2008), "Una introducción conceptual al desarrollo financiero, capital social y anonimidad: el caso de México", Documento de Trabajo DTE 427, México, CIDE, División de Economía.

Diario Oficial de la Federación (2005-2014): 1Dic2005, 17Feb2006, 2Jun2006, 3Jul2006, 23Ago2006, 25Ago2006, 31Oct2006, 11Ene2007, 1Feb2007, 23May2007, 21Jun2007, 16Jul2007, 6Sep2007, 24Sep2007, 22Ene2007, 20Dic2007, 30Ene2008, 14Mar2008, 16Abr2008, 21Abr2008, 24Abr2008, 12May2008, 13Jun2008, 27Jun2008, 1Ago2008, 29Ago2008, 9Sep2008, 22Oct2008, 14Nov2008, 19Dic2008, 1Jul2009, 14Mar2008, 15Abr2010, 21Ene2011, 18Feb2011, 23Jun2011, 12Jul2011, 26Jul2011, 24Ene2012, 9Feb2012, 14Jun2012, 1Ago2012, 8Ago2012, 10Oct2012, 30Nov2012, 5Dic2012, 31Dic2012, 2Abr2013, 4Abr2013, 11Jul2013, 20Ago2013, 24Abr2013, 19Jun2013, 15Jul2013, 17Jul2013, 20Ago2013, 11Sep2013, 13Sep2013, 6Dic2013, 9Dic2013, 10Dic2013, 12Mar2014, 24Mar2014, 29Abr2014, 30Abr2014, 18Jun2014, 29Ago2014, 23Dic2014. México

Estados Financieros (2008-2013): GlobalCard, 2008. Vivir Soluciones Financieras, 2008. Volkswagen Bank, 2008. Servicios Financieros Soriana, 2011, 2012. Banco Santander, 2011. Banorte, 2012. Scotiabank Inverlat, 2012. Ve por Más, 2012. Ixe, 2012. Banobras, 2013, México

García, Jannet (2009), Valuación de BORHIS: Modelo de Prepago, tesis de maestría, México, Cimat.

Guerrero, Rodolfo y Villalpando, Mario (2009), "Rentabilidad, concentración y eficiencia en el sistema bancario mexicano", El Trimestre Económico, vol. LXXVI, núm. 301, pp. 237-263. 
Hernández, Fausto (2010), "Obstáculos al desarrollo del sistema financiero en México", Documento de Trabajo DTE 484, México, CIDE, División de Economía.

INEGI (2012), Encuesta Nacional de Inclusión Financiera, México, [en línea], disponible en: http://www.inegi.org.mx/est/contenidos/Proyectos/encuestas/hogares/especiales/enif/enif2012/default.aspx. [Consultado el 30 de diciembre de 2014].

Kemeny, John y Snell, J. Lurie (1976), Finite Markov Chains, Nueva York, Springer.

Poder Ejecutivo Federal, Gobierno de los Estados Unidos Mexicanos, Presidencia de la República (2013), Plan Nacional de Desarrollo 2013-2018.

Poder Ejecutivo Federal, Gobierno de los Estados Unidos Mexicanos, Presidencia de la República, (2007) Plan Nacional de Desarrollo 2007-2012.

Ramírez, Mauricio (2000), "Competencia en el mercado de crédito de la Banca Múltiple en México: un análisis a través de procesos de Markov con carteras desagregadas por calificación de riesgo", tesis de maestría, México, CIDE.

Rivera, Eugenio y Rodríguez Adolfo (2007), Competencia y regulación en la banca de Centroamérica y México: Un estudio comparativo, México, Cepal, Publicación de Naciones Unidas.

Rojas, Mariano (1997), "Competencia por clientes en la industria bancaria de México", El Trimestre Económico, vol. LXIV (1), núm. 253, pp. 47-73. Rodríguez, Eduardo (2003), "Concentración industrial y rentabilidad de la banca en México: Evaluación posterior a la crisis de 1995", El Trimestre Económico, vol. LXX, núm. 278, pp. 371-404.

Ross, Sheldon M. (1996), Stochastic Processes. 2 ${ }^{\text {a. }}$ ed., John Wiley y Sons, Inc. SHCP (2006), Guía para la Constitución de una Sociedad Financiera de Objeto Múltiple, México, [en línea], disponible en: http://www.hacienda.gob. $\mathrm{mx} /$ sitios_interes/otros_sitios/sofomes/guiaparaconstitucion_sofomes. pdf. [Consultado el 30 de diciembre de 2014].

\section{APÉNDICE}

Ajuste relacionado con la información de las Sofom ENR

Dado que la Condusef $(2013,2014)$ reporta que 48 por ciento de las Sofom ENR no están operando o no han registrado contratos de adhesión de los productos y servi- 
166 ECONOMÍA TEORÍA Y PRÁCTICA • Nueva Época, número 47, julio-diciembre 2017

cios financieros que ofertan en el mercado; $K N$ y $N N$ se redujeron al 52 por ciento, en las tablas 4.1 y 4.3 y se obtuvo nuevamente la matriz $P$ de probabilidades de transición:

Tabla A1. Matriz de transición 2012-2013

(Ajustando el número de Sofom enr al 52 por ciento)

\begin{tabular}{|c|c|c|c|c|c|c|c|c|c|}
\hline \multicolumn{2}{|c|}{} & \multicolumn{9}{|c|}{2013} \\
\cline { 2 - 10 } \multicolumn{2}{|c|}{} & $N$ & $R$ & $U$ & $C$ & $F$ & $B$ & $M$ & $K$ \\
\hline \multirow{5}{*}{$\approx$} & 0.9986 & 0 & 0 & 0 & 0.0007 & 0 & 0.0007 & 0 \\
\hline & $R$ & 0 & 0.88 & 0 & 0 & 0 & 0 & 0 & 0.12 \\
\hline & $U$ & 0 & 0 & 0.983 & 0 & 0 & 0 & 0 & 0.017 \\
\cline { 2 - 10 } & $C$ & 0 & 0 & 0 & 0.986 & 0 & 0 & 0 & 0.014 \\
\hline & $F$ & 0 & 0 & 0 & 0 & 1 & 0 & 0 & 0 \\
\hline & $B$ & 0 & 0 & 0 & 0 & 0 & 1 & 0 & 0 \\
\hline & $M$ & 0 & 0 & 0 & 0 & 0 & 0 & 0.952 & 0.048 \\
\hline & $K$ & 0.902 & 0.007 & 0.002 & 0.082 & 0.002 & 0.003 & 0.002 & 0 \\
\hline
\end{tabular}

Fuente: Elaboración propia con datos de la CNBV y de la Condusef.

Tabla A2. Matriz de transición 2013-2014

\begin{tabular}{|c|c|c|c|c|c|c|c|c|c|}
\hline & \multicolumn{8}{|c|}{2014} \\
\hline & & $\mathrm{N}$ & $R$ & U & c & $\mathrm{F}$ & B & M & K \\
\hline \multirow{8}{*}{$\stackrel{m}{\bar{\sigma}}$} & $\mathrm{N}$ & 0.9972 & 0.0007 & 0 & 0 & 0.0014 & 0 & 0.0007 & 0 \\
\hline & $R$ & 0 & 0.88 & 0 & 0 & 0 & 0 & 0 & 0.1200 \\
\hline & U & 0 & 0 & 0.8655 & 0 & 0 & 0 & 0 & 0.1345 \\
\hline & C & 0 & 0 & 0 & 0.9855 & 0 & 0 & 0 & 0.0145 \\
\hline & F & 0 & 0 & 0 & 0 & 1 & 0 & 0 & 0 \\
\hline & $B$ & 0 & 0 & 0 & 0 & 0 & 1 & 0 & 0 \\
\hline & M & 0 & 0 & 0 & 0 & 0 & 0 & 0.9524 & 0.0476 \\
\hline & K & 0.8791 & 0.0066 & 0.0017 & 0.1043 & 0.0033 & 0.0033 & 0.0017 & 0 \\
\hline
\end{tabular}

Fuente: Elaboración propia con datos de la CNBV y de la Condusef. 